\title{
Radiobiological Studies with Monoenergetic Neutrons ${ }^{1}$
}

\author{
E. J. Hall, H. H. Rossi, A. M. Kellerer, \\ L. Goodman, and S. Marino \\ Radiological Research Laboratory, Department of Radiology, College of Physicians \\ and Surgeons, Columbia University, New York, New York 10032
}

Hall, E. J., Rossi, H. H., Kellerer, A. M., Goodman, L., and Marino, S. Radiobiological Studies with Monoenergetic Neutrons. Radiat. Res. 54, 431-443 (1973).

The Radiological Research Accelerator Facility (RARAF) has the capability of producing essentially monoenergetic neutron beams, ranging in energy from $15.4 \mathrm{MeV}$ down to $220 \mathrm{keV}$. In addition, two lower energy neutron beams are available which consist of a wide spectrum of energies and are described as the $110 \mathrm{keV}$ and $60 \mathrm{keV}$ spectra. Seedlings of Vicia faba have been used to measure the oxygen enhancement ratio (OER) and the relative biological effectiveness (RBE) of each of these neutron beams. The OER decreases as the neutron energy is reduced between $15.4 \mathrm{MeV}$ and $220 \mathrm{keV}$, but does not appear to decrease further for lower energy neutrons. RBE increases as the neutron energy is reduced from $15.4 \mathrm{MeV}$ to $440 \mathrm{keV}$; the curve then goes through a maximum at around $350 \mathrm{keV}$, and for lower energies the $\mathrm{RBE}$ falls again.

\section{INTRODUCTION}

The Radiological Research Accelerator Facility (RARAF) is a joint enterprise between the Radiological Research Laboratory of Columbia University and the Medical Research Center of the Brookhaven National Laboratory. It consists of a large Van de Graaff accelerator, capable of accelerating protons or deuterons up to an energy of $4.2 \mathrm{MeV}$. By using suitable targets, essentially monoenergetic neutron beams can be generated, ranging in energy from $15.4 \mathrm{MeV}$ down to 220 $\mathrm{keV}$; in addition, two lower energy neutron beams are available which consist of a wide spectrum of energies and are described as the $110 \mathrm{keV}$ and the $60 \mathrm{keV}$ spectra.

This unique facility has been used by a number of collaborators, and radiobiological findings have been reported for several neutron energies (1-3). The present paper describes a project in which the oxygen enhancement ratio (OER) and the relative biological effectiveness (RBE) were studied over the entire range of neutron energies available at RARAF.

The radiobilogical test system chosen for this investigation was the inhibition of root growth of seedlings of Vicia faba, the techniques for which have been well

${ }^{1}$ This investigation was supported by Contract AT-(11-1)-3243 for the U. S. Atomic Energy Commission and Public Health Service Research Grant CA-12536-01 from the National Cancer Institute. 
established over a period of years (4-6). This particular biological material was chosen for two reasons. First, the dose rates available from the machine, particularly at lower energies, are limited, and consequently it is necessary to use a biological material which is relatively radiosensitive and does not require large doses. Second, in order to obtain an oxygen enhancement ratio in a situation where the dose rate is limited, it is necessary to maintain specimens under conditions of hypoxia for prolonged periods of time. While this is technically possible with plant seedlings, it involves insuperable difficulties with more fragile biological material such as mammalian cells in culture.

TABLE I

Composition of Culture Medium

\begin{tabular}{llc}
\hline \multicolumn{1}{c}{ Material } & \multicolumn{1}{c}{ Chemical symbol } & $\begin{array}{c}\text { Concentration } \\
\text { g/1000 liter }\end{array}$ \\
\hline Ferrous ammonium sulfate & $\mathrm{Fe}\left(\mathrm{NH}_{4}\right)_{2} \mathrm{SO}_{4} \cdot 6 \mathrm{H}_{2} \mathrm{O}$ & 42.0 \\
Manganese sulfate & $\mathrm{Mn} \mathrm{SO}_{4} \cdot \mathrm{H}_{2} \mathrm{O}$ & 15.5 \\
Zinc sulfate & $\mathrm{Zn} \mathrm{SO}_{4} \cdot 7 \mathrm{H}_{2} \mathrm{O}$ & 22.0 \\
Ammonium molybdate & $\left.\mathrm{NH}_{4}\right)_{2} \mathrm{MO}_{7} \mathrm{O}_{24} 7 \mathrm{H}_{2} \mathrm{O}$ & 3.6 \\
Cupric sulfate & $\mathrm{Cu} \mathrm{SO}_{4}$ anhydrous & 1.0 \\
Ammonium vanadate & $\mathrm{NH}_{4} \mathrm{VO}_{3}$ & 0.46 \\
Cobalt sulfate & $\mathrm{Co} \mathrm{SO}_{4} \cdot 7 \mathrm{H}_{2} \mathrm{O}$ & 0.48 \\
Boric acid & $\mathrm{H}_{3} \mathrm{BO}_{3}$ & 0.57 \\
Nickel sulfate & $\mathrm{Ni} \mathrm{SO}_{4} \cdot 6 \mathrm{H}_{2} \mathrm{O}$ & 0.45 \\
Chromium potassium sulfate & ${\mathrm{Cr} \mathrm{K}\left(\mathrm{SO}_{4}\right)_{2} \cdot 12 \mathrm{H}_{2} \mathrm{O}}_{0.96}$ & 100.0 \\
Ammonium nitrate & $\mathrm{NH}_{4} \mathrm{NO}_{3}$ & 100.0 \\
Potassium hypophosphate & $\mathrm{K} \mathrm{H}_{2} \mathrm{PO}_{4}$ & 80.0 \\
Calcium carbonate & $\mathrm{Ca} \mathrm{CO}_{3}$ & 100.0 \\
Magnesium sulfate & $\mathrm{Mg} \mathrm{SO}_{4} \cdot 7 \mathrm{H}_{2} \mathrm{O}$ & 100.0 \\
Nitrilotriacetic acid & & \\
\hline
\end{tabular}

\section{MATERIALS AND METHODS}

\section{Culture of the Seedlings}

In each experiment, about 500 seeds were soaked in running water for 3 days. Those that had germinated were planted in moist vermiculite, a medium used commercially to raise seedlings, where they were left for 5 days. By this time the primary root of each seedling was approximately $10 \mathrm{~cm}$ in length, at which time they were transferred to the main culture tank, and placed on a lucite lid with their roots pointing downward into the culture medium maintained at $19^{\circ} \mathrm{C}$. The culture medium consists of a solution of salts and trace elements, the composition of which is shown in Table I.

The seedlings were sorted into groups carefully matched so that the distribution of lengths within each group was similar. The number of seedlings per group was 12 in all experiments except those involving the 2 lowest energy neutron spectra, and in this case only 6 roots per group were used. The seedlings to be irradiated and their controls, were transported the 60 miles from Columbia University to the Brookhaven National Laboratory in lucite containers which were insulated 
to minimize temperature fluctuations, and in which vigorous aeration was maintained at all times. The details of the culture procedure have been reported previously $(6)$.

The irradiations were carried out in specially constructed lucite fixtures, designed to accommodate the seedlings in such a way that the sensitive root tips came together into a small volume. The dimensions of this critical volume varied with the energy of neutrons involved; this will be discussed further in the next section. The lid of each fixture was screwed down tightly onto a rubber O-ring to effect a seal. The seedlings were aerated or rendered hypoxic by bubbling a stream of air or high purity nitrogen at the flow rate of 1 liter/min through the water into which the primary roots of the seedlings were immersed. In the latter case, the gas was flushed through the system for 30 min prior to the irradiation, which was shown in previous experiments to be adequate to render the roots hypoxic $(7,8)$.

Prior to irradiation the length of each primary root was measured from a reference point on the hypocotyl to the growing tip. Roots were cultured after irradiation for a period of 10 days at $19^{\circ} \mathrm{C}$, and at the end of this time each root was remeasured and the growth increment during the 10-day period calculated. The mean 10-day increment for each group, divided by the corresponding quantity for control roots, is termed "growth in 10 days." This quantity is a measure of the radiation damage in the root meristem, and has been used for many years $(7,9,10)$.

\section{Methods of Irradiation}

The irradiations were carried out at RARAF with neutron energies of 15.4, 6,2 , and $1 \mathrm{MeV}, 440,340$, and $220 \mathrm{keV}$. In addition, two neutron spectra with a nominal maximum energy of $110 \mathrm{keV}$ and of $60 \mathrm{keV}$ were used. For comparison, experiments were also performed with $210-\mathrm{kV}$ x-rays.

The seedlings were irradiated in lucite fixtures, the dimensions of which varied with the neutron energy. For $220 \mathrm{keV}$ and higher, the root tips were confined to an area $3 \times 3 \mathrm{~cm}$ in cross-section and restricted to a thickness of $0.3 \mathrm{~cm}$ in the direction of the beam. The fixture was mounted to locate the tip center plane at a distance of $10 \mathrm{~cm}$ from the neutron producing target. For $430 \mathrm{keV}$ and above the root tips were located at a depth of $0.8 \mathrm{~cm}$ of tissue-like material (lucite, tissue equivalent plastic, water, and bean root). For $220 \mathrm{keV}$ the tip depth was about $0.5 \mathrm{~cm}$.

For the two low energy neutron spectra, a smaller fixture was constructed such that the root tips were confined to an area $1 \mathrm{~cm}$ square at right angles to the direction of the beam, and $0.2 \mathrm{~cm}$ wide in the direction of the beam. The center plane of the root tips was then located at a depth of $0.18 \mathrm{~cm}$ and at a distance of $3.2 \mathrm{~cm}$ from the target.

Dose measurements for these neutron energies were performed with a discshaped tissue-equivalent ionization chamber. Depth dose measurements were made by the external addition of tissue equivalent plastic dises to the thin face of the chamber. The curves determined are shown in Fig. 1, which also indicates 


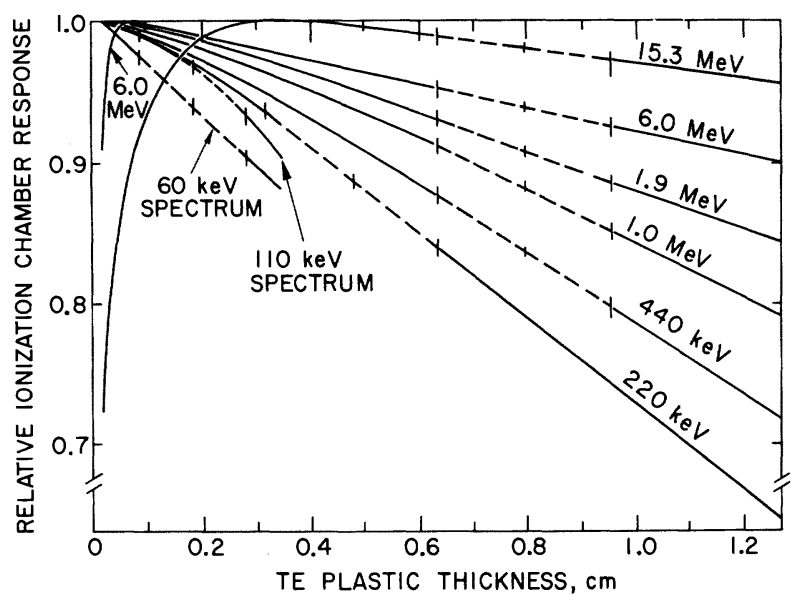

Fig. 1. Dose attenuation in tissue-equivalent plastic for various neutron energies. The broken lines between vertical bars indicate the size and position of the biological specimens during the rradiations at each neutron energy.

the secondary particle equilibrium build-up observed for the two highest energies. This figure also indicates the size and position of the biological specimen relative to the depth dose curve. For the dosimetry of the two low-energy spectra the small size and enhanced response of a spherical quarter-inch diameter tissue equivalent gas multiplication ionization chamber was utilized. Depth dose data were obtained by the external addition of nylon caps of different wall thickness. These curves are included in Fig. 1. Table II is a summary of the energies that were used, together with the range of energies involved, and the average dose rates in the various experiments.

TABLE II

\begin{tabular}{|c|c|c|c|c|c|c|c|}
\hline $\begin{array}{c}\text { Mean neutron } \\
\text { energy }\end{array}$ & $\begin{array}{l}\text { Energy } \\
\text { spread }\end{array}$ & $\begin{array}{l}\text { Nuclear } \\
\text { reaction }\end{array}$ & $\begin{array}{c}\text { Distance } \\
\text { from } \\
\text { target }\end{array}$ & Angle & $\begin{array}{c}\text { Gamma } \\
\text { contami- } \\
\text { nation } \\
\%\end{array}$ & $\begin{array}{c}\text { Dose } \\
\text { spread } \\
\text { through } \\
\text { specimen } \\
\%\end{array}$ & $\begin{array}{l}\text { Average } \\
\text { dose } \\
\text { rate } \\
\text { rads } / h r\end{array}$ \\
\hline $15.4 \mathrm{MeV}$ & $\pm 4 \%$ & ${ }^{3} \mathrm{H}\left({ }^{2} \mathrm{H}, \mathrm{n}\right){ }^{4} \mathrm{He}$ & $10 \mathrm{~cm}$ & $0^{\circ}$ & 3 & \pm 1 & 280 \\
\hline $6 \mathrm{MeV}$ & $\pm 7 \%$ & ${ }^{2} \mathrm{H}\left({ }^{2} \mathrm{H}, \mathrm{n}\right)^{3} \mathrm{He}$ & 10 & $0^{\circ}$ & 3 & \pm 3 & 450 \\
\hline $2 \mathrm{MeV}$ & $\pm 5 \%$ & ${ }^{3} \mathrm{H}\left({ }^{1} \mathrm{H}, \mathrm{n}\right)^{3} \mathrm{He}$ & 10 & $0^{\circ}$ & 5 & \pm 3 & 140 \\
\hline $1 \mathrm{MeV}$ & $\pm 11 \%$ & ${ }^{3} \mathrm{H}\left({ }^{1} \mathrm{H}, \mathrm{n}\right){ }^{3} \mathrm{He}$ & 10 & $0^{\circ}$ & 1 & \pm 2.5 & 70 \\
\hline $440 \mathrm{keV}$ & $\begin{array}{l}+22 \% \\
-18\end{array}$ & ${ }^{3} \mathrm{H}\left({ }^{1} \mathrm{H}, \mathrm{n}\right)^{3} \mathrm{He}$ & 10 & $100^{\circ}$ & 2 & \pm 4.5 & 40 \\
\hline $340 \mathrm{keV}$ & $\begin{array}{l}+28 \% \\
-23\end{array}$ & ${ }^{3} \mathrm{H}\left({ }^{1} \mathrm{H}, \mathrm{n}\right)^{3} \mathrm{He}$ & 10 & $120^{\circ}$ & 2 & \pm 5 & 30 \\
\hline $220 \mathrm{keV}$ & $\pm 30 \%$ & ${ }^{3} \mathrm{H}\left({ }^{1} \mathrm{H}, \mathrm{n}\right)^{3} \mathrm{He}$ & 10 & $120^{\circ}$ & 1 & \pm 16 & 16 \\
\hline $110 \mathrm{keV}$ spectrum & $110 \rightarrow$ zero & ${ }^{3} \mathrm{H}\left({ }^{1} \mathrm{H}, \mathrm{n}\right){ }^{3} \mathrm{He}$ & 3.2 & $100^{\circ}$ & 1 & \pm 10 & 35 \\
\hline $60 \mathrm{keV}$ spectrum & $60 \rightarrow$ zero & ${ }^{3} \mathrm{H}\left({ }^{1} \mathrm{H}, \mathrm{n}\right)^{3} \mathrm{He}$ & 3.18 & $100^{\circ}$ & 3 & \pm 15 & 15 \\
\hline
\end{tabular}


From the table it is evident that the dose rate varied appreciably from one neutron energy to another. In general, dose rate decreased with neutron energy to a point where exposure times approached several hours for the largest doses at the lowest energy. Nevertheless, a dose-rate effect, i.e., a change of biological effect with dose rate, would not be expected under these circumstances; there are two reasons for this. First, the low-energy neutrons, for which the dose rate is minimal, involve high LET events and result in dose-response relationships which do not significantly deviate from exponential functions; a dose-rate effect is usually associated with survival curves having an initial shoulder resulting from the accumulation of sublethal radiation damage. Second, Vicia seedlings are grown and irradiated at close to room temperature $\left(19^{\circ} \mathrm{C}\right)$ and do not exhibit a dramatic dose-rate effect, comparable with mammalian cells, even for low LET radiations over the range of dose rates involved.

\section{RESULTS}

Results obtained for the 9 different neutron energies are shown in Fig. 2. The experiments were performed over a period of more than a year. X-ray experiments were performed at the beginning, at the end, and half way through this period to insure that there was no systematic change in the radiation response of the system. The x-ray results are all pooled in Fig. 3.

The experimental endpoint is the growth of the primary root in ten days, $G$. The dose effect curves are given in plots of the logarithm of the growth relative to controls, $G / G_{0}$, versus dose, $D$. For the neutron experiments least square fits to exponential functions are inserted:

$$
G=G_{0} e^{-a D}
$$

The treatment is in accordance with the normal usage in the analysis of dose effect curves, but it is a departure from the earlier approach chosen for the analysis of the Vicia faba data $(7,9,10)$. In the earlier approach plots of $G$ vs $\ln D$ were used and straight lines in this plot were chosen for least square fitting. This corresponds to the equation,

$$
G=G_{0}-s \ln (c D)
$$

The two approaches are not mutually exclusive because for the values of $s$ which have been experimentally observed, Eq. (2) results in curves which are very near to an exponential function for $G / G_{0}$ from 0.8 to 0.2 .

In the earlier analysis, Eq. (2) has been chosen because multiplication of the dose by a constant factor then merely corresponds to a parallel shift of the straight line in the $G$ vs $\ln D$ plot. This reduces the statistical analysis of OER to a parallel line assay. In the present study, the statistical method for the derivation of OER has been retained and the resulting OER values will be given further below. With the exception of this special application the representation of the $\ln G$ versus $D$ has been used as the basis of the present analysis; this is not only in line with the more common treatment of dose effect curves but also eliminates the difficulty which arises from the fact that Eq. (2) diverges at low doses. 


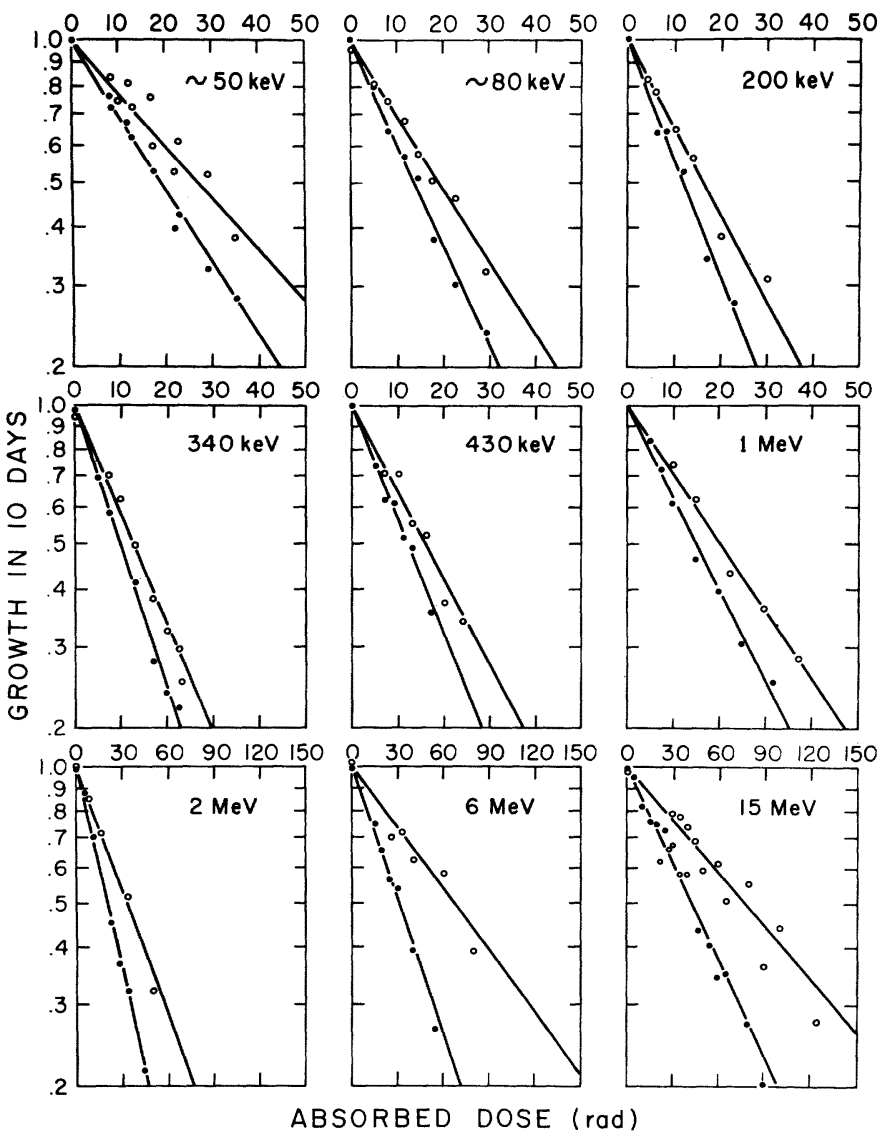

Fig. 2. Growth of bean roots in 10 days relative to that of controls as a function of dose for various neutron energies. Full circles refer to aerated conditions, open circles to hypoxic conditions. The lines result from least square fits of Eq. (5) to the properly weighted data points.

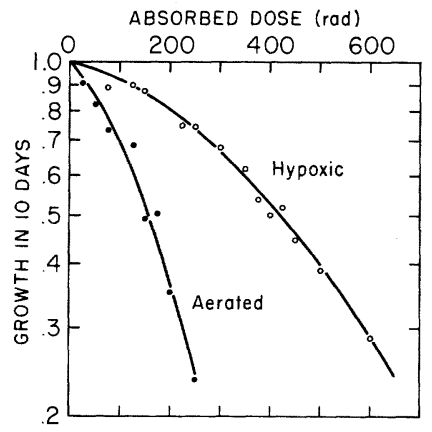

Fig. 3. Growth of bean roots in 10 days relative to that of controls as a function of X-ray dose. Full circles refer to aerated conditions, open circles to hypoxic conditions. The lines result from least square fits of Eq. (3) to the properly weighted data points. 
According to the theory of dual radiation action $(11,12)$ the elementary lesions underlying a wide range of cellular radiation effects increase with the square of the specific energy in sites comparable to, but somewhat smaller than, the nuclear volume. This leads to a linear-quadratic dependence on dose; the linear component represents the interaction of lesions produced in one and the same particle track while the quadratic component stands for that part of the effect which is due to the interaction of lesions produced by independent particle tracks $(11,12)$. In the case of neutrons the linear component dominates in the range of small and intermediate doses, as evidenced by the frequently observed exponential dose-effect relations. For x-rays, on the other hand, the quadratic component dominates except at very small doses. In the general case, one must account for the possibility that both for sparsely and densely ionizing radiations one may

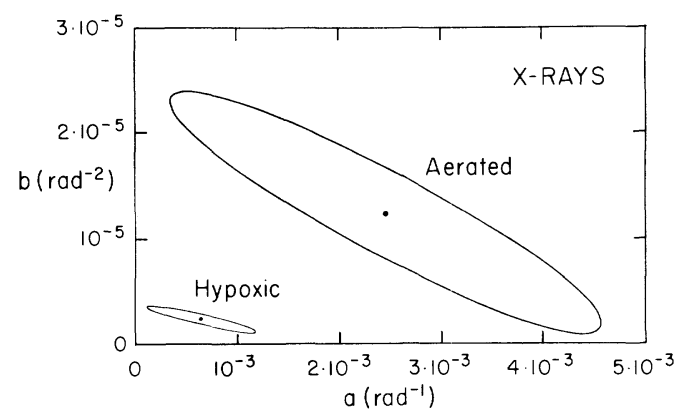

Fig. 4. Least square estimates and $95 \%$ confidence regions for the parameter $a$ and $b$ in Eq. (3).

deal with a linear as well as a quadratic component in dose. Accordingly, a leastsquare fit of the experimental data both for x-rays and the various neutron energies has been performed for the logistic equation,

$$
G=G_{0} e^{-a D-b D^{2}} .
$$

Linear regression techniques can be applied for this purpose because the logarithm of $G$ depends linearly on the three parameters, $\ln G_{0}, a$, and $b$ :

$$
\ln G=\ln G_{0}-a D-b D^{2}
$$

In addition to the least-square estimates of the parameters, the joint $95 \%$ confidence regions for the parameters have been determined on the basis of Fisher's F distribution (for details of the linear regression analysis see for example (19)). The analysis has been based on the complete data of all individual roots and not merely on the mean values obtained for different dose values in a given experiment.

For the pooled $\mathrm{x}$-ray data, representing a total of approximately 500 roots, the estimates $a=2.5 \times 10^{-3}\left(\mathrm{rad}^{-1}\right)$ and $b=1.2 \times 10^{-5}\left(\mathrm{rad}^{-2}\right)$ have been obtained for the experiments in air, and the values $a=0.63 \times 10^{-3}\left(\mathrm{rad}^{-1}\right)$ and $b=0.25$ $\times 10^{-5}\left(\mathrm{rad}^{-2}\right)$ have been obtained for the hypoxia experiments. The $95 \%$ confidence regions for the parameters $a$ and $b$, as indicated in Fig. 4, are wide, but it is important to note that both parameters are strongly correlated. It is therefore decisive to give the joint confidence intervals and not merely, as is 
commonly done, confidence intervals for the individual parameters. In the following the estimated values of the parameters will be used for comparison with the results of the neutron experiments.

In the analysis of the neutron data, it has been found that the resulting confidence ranges for $b$ are very broad. This is to be expected because for neutrons the linear component in Eq. (4) is in all cases dominant over the quadratic component, and the least-square fit is therefore not significantly affected by the quadratic term. Accordingly, there is no indication that the quadratic component is different in the case of neutrons from that observed for x-rays. Since, however, the contribution of the quadratic term is negligible the simple fit to an exponential function with only two free parameters $G_{0}$ and $a$ has been applied for all neutron data:

$$
G=G_{0} e^{-a D}
$$

and the resulting curves have been inserted in Fig. 2.

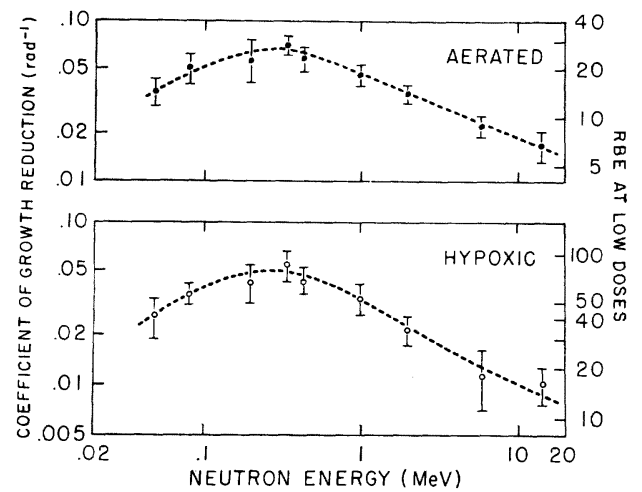

FIG. 5. Least square estimates of the parameter $a$ in Eq. (5) and the corresponding $95 \%$ confidence ranges for various neutron energies. The broken lines are inserted to show the trend of the results; they have no mathematical significance.

The estimate and $95 \%$ confidence regions of the parameter $a$ are given in Fig. 5 for various neutron energies. The broken line merely serves to show the trend of the data, it has no mathematical significance.

The RBE at low doses is equal to $a_{x} / a_{n}$, where $a_{x}$ and $a_{n}$ are the linear coefficients for $x$-rays and neutrons. The values of RBE as a function of neutron energy are given on the right ordinate scale of Fig. 5. The confidence intervals are based solely on the regression analysis of the neutron data and on the estimated value of the parameter $a_{x}$ for x-rays; the uncertainty in the value of $a_{x}$ is disregarded. According to the confidence regions in Fig. 4 the curves of RBE could therefore be shifted with regard to the RBE scale. The shape of the curves in this logarithmic representation is, however, unaffected by such a shift. Moreover one obtains the same curve shape if one considers the RBE at a certain effect level, instead of the extrapolated value of $R B E$ at very low doses; this merely introduces a constant factor in the RBE values as can be seen in the following way. From Eq. (3) for x-rays and Eq. (5) for neutrons one has the 
following relation for the $\mathrm{RBE}$ of neutrons:

$$
\mathrm{RBE}=a_{n} /\left(a_{x}+b_{x} D_{x}\right) .
$$

Thus RBE is always proportional to $a_{n}$, while the factor $\left(a_{x}+b_{x} D_{x}\right)^{-1}$ which connects the two quantities RBE and $a_{n}$ decreases with increasing effect level. For any chosen effect level the value $D_{x}$ is read off Fig. 3, or is calculated from Eq. (3) and the estimated parameters $a_{x}$ and $b_{x}$. In the aerated case one obtains

$$
\mathrm{RBE}=a_{n} / a_{x}=400 a_{n}
$$

for very small doses (see right ordinate scale in Fig. 5), while for $50 \%$ growth reduction one has $D_{x}=160$ and accordingly,

$$
\mathrm{RBE}=a_{n} /\left(a_{x}+l_{x} D_{x}\right)=226 a_{n}
$$

From the broken lines in Fig. 5, an estimate of the dependence of the oxygen enhancement ratio (OER) on neutron energy can be obtained. This estimate is given as a broken line in Fig. 6, it has, however, as little precise mathematical

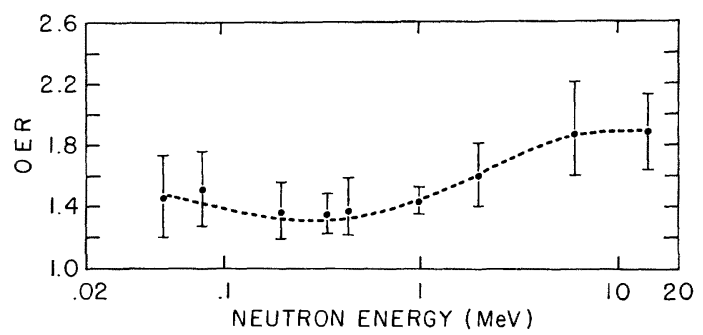

Fig. 6. Least-square estimates of the oxygen-enhancement ratio (OER) and the corresponding 95\% confidence ranges for various neutron energies. The broken line corresponds to the two broken lines in Fig. 5 .

meaning as the broken lines in Fig. 5, and it remains, therefore, to obtain the actual confidence intervals for the values of OER. Such confidence limits cannot readily be obtained from the confidence ranges of the $\mathrm{RBE}$ values. This is due to the fact that the experiment in air and nitrogen at a given neutron energy share the same control group. The estimates of the two parameters $a_{\mathrm{Air}}$ and $a_{\mathrm{N} \text { itrogen }}$ are therefore not independent and the confidence range for OER $=a_{\mathrm{A} \text { ir }} / a_{\mathrm{N} \text { itrogen }}$ cannot be obtained directly from the two confidence ranges for $a_{\mathrm{Air}}$ and $a_{\mathrm{Nitrogen}}$. For this reason the confidence ranges for OER are derived by a parallel line assay according to Eq. (2). The results are superimposed to the broken line given in Fig. 6. Application of this particular statistical test has the additional advantage that one obtains a cross check between the results based on two different analytical approximations of the data. One finds that the results are in sufficient agreement. Although the $95 \%$ confidence ranges for the values of OER are broad, there is a clear indication of an increase of OER with increasing neutron energy. Whether the OER also increases when the energy of the neutrons falls below $100 \mathrm{kV}$ cannot be decided on the basis of the present data. It should be pointed out that the neutrons have been essentially monochromatic for higher energies but that for 
the two lowest energies one is dealing with a broad spectrum ranging from zero energy up to $110 \mathrm{kV}$ in the one case, and from zero energy up to $60 \mathrm{kV}$ in the other case. The energies of $80 \mathrm{kV}$ and $50 \mathrm{kV}$ have been taken as rough estimates of the effective energy in each case.

It should be noted that one cannot a priori assume that for each radiation quality the OER is a constant, independent of dose. In the present experiments with neutrons a dependence of OER on dose can, however, not be detected because both under oxygenated and hypoxic conditions the dose-response curves do not significantly differ from exponential functions; if all response curves are exponen-
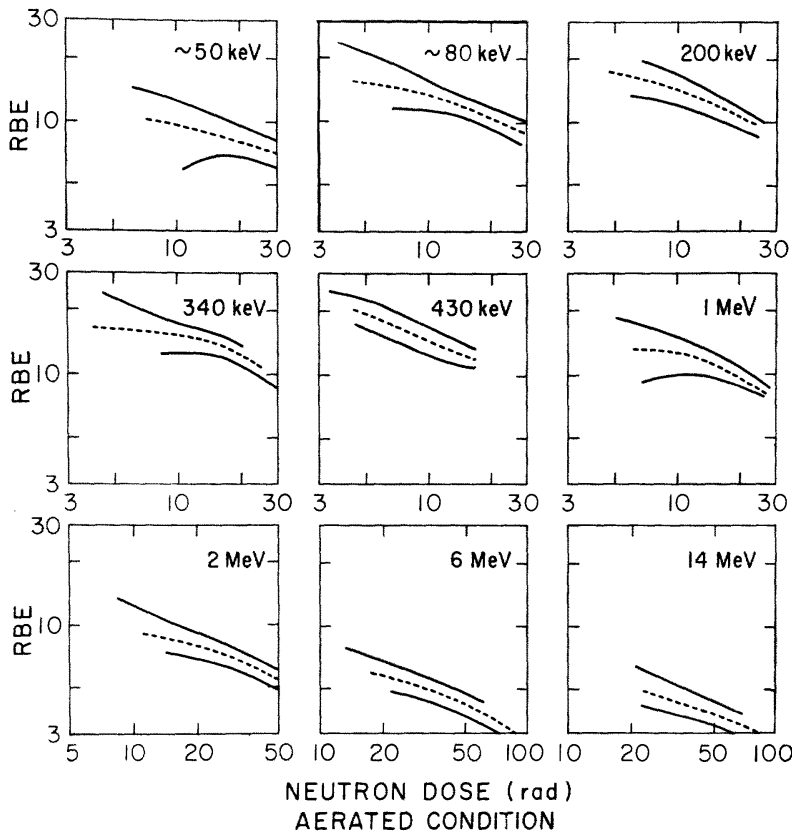

FIG. 7. Estimates (broken lines) of the dose-RBE dependence and the $95 \%$ confidence regions (solid lines) for various neutron energies under aerated conditions.

tial the OER is independent of dose. As an additional check, however, a more direct statistical test has been employed which is not based on the choice of a particular equation for the response curves. The test consists of a direct nonparametric comparison of each dose given under oxygenated conditions with each dose given under hypoxic conditions. This analysis, details of which are described in a separate paper (14), is applicable both to the evaluation of OER and of RBE as a function of dose. Results for the RBE analysis will be given below; as far as the OER values are concerned, it is sufficient to state that the results are in good agreement with the results represented in Fig. 6, and that no dependence of OER on dose can be detected in the present experiments. This does, of course, not exclude a possible dependence of OER on dose which might be revealed by a more extensive series of experiments, or in an experimental system where the 
response can be studied over a wider range than in the growth reduction studies of Vicia faba.

For the RBE of neutrons a strong dependence on dose has been shown in various systems. Such a dependence is also found in the present study. Figs. 7 and 8 give the confidence regions and the statistical estimates of RBE as a function of neutron dose. These results are also obtained by the nonparametric test which has been applied earlier (15) to an analysis of the RBE of neutrons in the opacification of the murine lens. One finds that at intermediate and higher effect levels the results are all consistent with the assumption that the curves in this double-logarithmic representation have a slope of -0.5 , as has been postulated in the theory of dual radiation action (11). At small doses the results
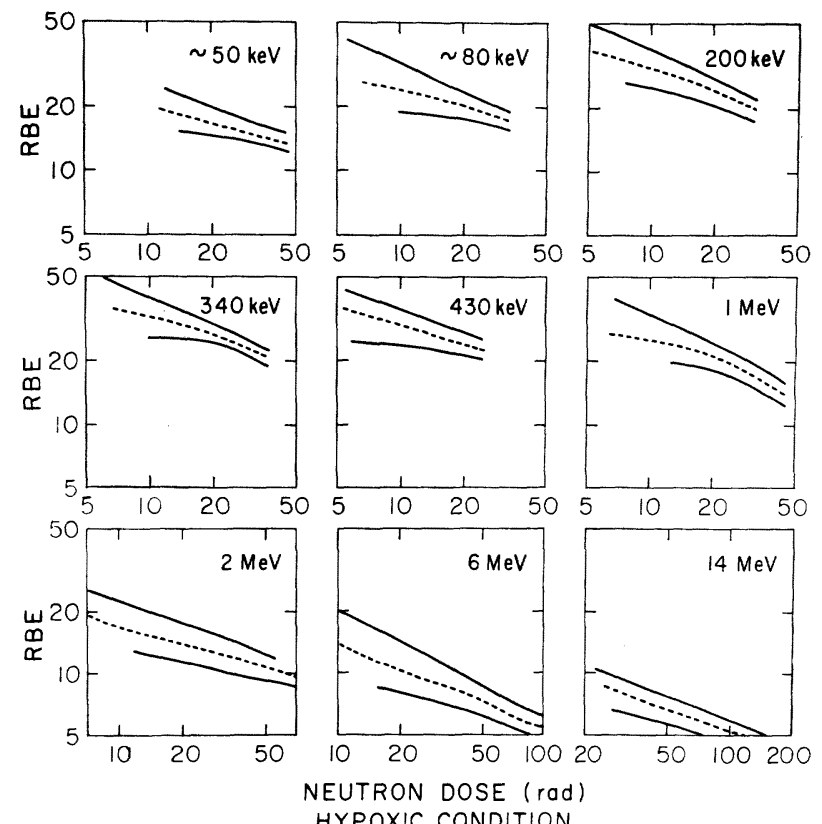

Fig. 8. Estimates (broken lines) of the dose-RBE dependence and the $95 \%$ confidence regions (solid lines) for various neutron energies under hypoxic conditions.

indicate a leveling out of $\mathrm{RBE}$, as is also expected on the basis of the theory. The confidence regions are, however, wide and consequently the shape of the $\mathrm{RBE}$ curves cannot be determined with great accuracy. One must also note that all curves relate to the same $\mathrm{x}$-ray data and that the results are therefore not statistically independent. Nevertheless the results are useful because they complement the results obtained from overall curve fitting; in Fig. 5 confidence limits are given which are valid only for the quantity $a_{n}$ and not for RBE. The results represented in Fig. 8 are also of interest insofar as they show that the direct statistical analysis of the dose dependence of RBE yields confidence intervals for RBE at intermediate and high doses which are even more narrow than the confidence intervals for the parameter $a_{n}$ obtained by curve fitting and which are given in Fig. 5. 


\section{DISCUSSION}

As can be seen from Fig. 6, the oxygen enhancement ratio decreases as the neutron energy is decreased between $15 \mathrm{MeV}$ and $220 \mathrm{keV}$. The data for the two low-energy spectra are less accurate than for the higher energies. This is a result of technical difficulties involved with these two low energies. Because of the low dose rates, a short working distance of only $3 \mathrm{~cm}$ was necessitated, and as a result only 6 seedlings per group could be irradiated. Consequently, the experimental fluctuations are greater than in the case of higher energies. This is at once evident from a cursory examination of the raw data presented in Fig. 2. As plotted in Fig. 6 the OER does not appear to decrease further below $220 \mathrm{keV}$, and indeed there is a possibility that it begins to rise again.

There are a number of other reports in the literature concerning the dependence of OER on neutron energy. The situation is very complex but some comparison with the results presented in this paper is clearly called for. The present data indicate a greater change of OER with neutron energy than was reported by Broerse, Barendsen and Van Kersen (16) using mammalian cells in culture over a range of neutron energies from 1 to $15 \mathrm{MeV}$.

Using P388 leukemia cells in vivo, Berry (17) found an increasing OER with neutron energy from 1 to $25 \mathrm{MeV}$. The increase from $6 \mathrm{MeV}$ upwards is quite similar to that reported here, but in striking contrast to the present data, and indeed contrary to other reports in the literature, Berry found a very low OER at $1 \mathrm{MeV}$. Too much importance cannot be ascribed to this difference since, not only were the biological systems totally different, but the $1 \mathrm{MeV}$ neutrons described in the present report are essentially monoenergetic whereas Berry used a spectrum of fission neutrons.

A further possible factor could be a dose dependence of OER, especially for 15 and $25 \mathrm{MeV}$ neutrons. At these high neutron energies and at small doses, the biological effect is determined mainly by heavy recoils and the densely ionizing parts of the proton tracks; this should result in a low OER. At higher doses, the quadratic component due to the sparsely ionizing tracks of the fast protons becomes more significant and consequently a higher OER might be expected. Kellerer and Rossi (12) have calculated the dependence of OER on neutron energy on the basis of microdosimetric data and of the cellular inactivation cross-sections obtained by Barendsen (18) for low doses of heavy charged particles. These calculations lead to a nearly constant OER from a neutron energy of 300 $\mathrm{keV}$ to $14 \mathrm{MeV}$ and are in essential agreement with the experimental results of Broese et al. (16).

Berry, on the other hand, has obtained large values of OER for neutrons of $25 \mathrm{MeV}$ in experimental studies which involve high doses of the order of $2000 \mathrm{rad}$. The dose range in the present study is intermediate and the moderate increase of OER at higher neutron energies may therefore reflect a partial contribution of the fast protons to the effect, although the statistical accuracy of the survival curves for 14- $\mathrm{MeV}$ neutrons is not sufficient to establish the quadratic component.

Dennis and Boot (19), scoring pink mutations in Tradescantia, found a rapid reduction in OER between $6 \mathrm{MeV}$ and $100 \mathrm{keV}$ which is in reasonable agreement with the results presented in this paper; however, two other features of their 
work were not observed here. They found that the OER decreased again as the neutron energy rose from $6 \mathrm{MeV}$ to $15 \mathrm{MeV}$, and they also found a large OER, in excess of 2 , for $400 \mathrm{keV}$ neutrons which they attributed to oxygen resonance; neither of these findings was confirmed in the present series of experiments.

Variation of RBE as a function of neutron energy shows a rapid increase of $\mathrm{RBE}$ as the neutron energy is reduced from $15 \mathrm{MeV}$ down to $440 \mathrm{keV}$. The curve then goes through a maximum at around $350 \mathrm{keV}$ and for lower energies the RBE falls again. The data are in substantial agreement with the dependence of RBE on neutron energy which has been predicted on the basis of microdosimetric data (12).

RECEIVED : November 2, 1972

\section{REFERENCES}

1. J. L. Bateman and M. R. Snead, Current research in neutron RBE in mouse lens opacity. Symposium on Neutrons in Radiobiology. Oak Ridge, Nov. 1969.

2. A. G. Underbrink and A. H. Sparrow, Power relations as an expression of relative biological effectiveness (RBE) in Tradescantia stamen hairs. Radiat. Res. 46, 580-587 (1971).

3. A. G. Underbrink, Rhoda C. Sparrow, A. H. Sparrow and H. H. Rossi, RBE values of $\mathrm{X}$ rays and $0.43 \mathrm{MeV}$ monoenergetic neutrons on somatic mutations and loss of reproductive integrity in Tradescantia stamen hairs. Radiat. Res. 44, 187-203 (1970).

4. H. J. Evans, G. J. Neary, and S. M. Tonkinson, The use of colchicine as an indicator of mitotic rate in broad bean root meristems. J. Genet. 55, 487-502 (1957).

5. J. READ. The Radiation Biology of Vicia faba. Blackwell Scientific Publications, Oxford, 1959.

6. E. J. HALL. The relative biological effectiveness of $\mathrm{X}$ rays generated at $220 \mathrm{kVp}$ and gamma rays from a cobalt 60 teletherapy unit. Brit. J. Radiol. 34, 313-317 (1961).

7. E. J. Hall and J. Cavanagh. The oxygen effect for acute and protracted radiation exposures measured with seedlings of Vicia faba. Brit. J. Radiol. 40, 128-133 (1967).

8. E. J. Hall and J. Cavanagh. The effect of hypoxia on recovery of sublethal damage in Vicia seedlings. Brit. J. Radiol. 42, 270-277 (1969).

9. J. READ. The effect of ionizing radiation on the broad bean root. Part X. Brit. J. Radiol. 25, 89-99 (1952).

10. E. J. Hall and L. G. Lajtha. The recovery of Vicia faba meristem cells from x-radiation. Radiat. Res. 20, 187-194 (1963).

11. A. M. Kellerer and H. H. Rossi. RBE and the primary mechanism of radiation action. Radiat. Res. 47, 15-34 (1971).

12. A. M. KELLERER and H. H. Rossi. The theory of dual radiation action. In Current Topics in Radiation Research (M. Ebert and A. Howard, Ed.) 8, 85-158 (1972).

13. N. R. Draper and H. S. Smith. Applied Regression Analysis, J. Wiley, New York, 1966.

14. A. M. Kellerer and J. H. Brenot. Non-parametric determination of modifying factors in radiation action. Radiat. Res. In press.

15. J. L. Bateman, H. H. Rossi, A. M. Kellerer, C. V. Robinson, and V. P. Bond. Dose dependence of fast neutron RBE for lens opacification in mice. Radiat. Res. 51, 381-390 (1972).

16. J. J. Broerse, G. W. Barendsen, and G. R. van Kersen. Survival of cultured human cells after irradiation with fast neutrons of different energies in hypoxic and oxygenated conditions. Int. J. Radiat. Biol. 13, 559-572 (1967).

17. R. J. Berry. Hypoxic protection against fast neutrons of different energies-A review. Europ. J. Cancer 7, 145-152 (1971).

18. G. W. Barendsen. Mechanism of action of different ionizing radiations on the proliferative capacity of mammalian cells. In Theoretical and Experimental Biophysics. (A. Cole, Ed.), Vol. 1, 167-231. Marcel Dekker, New York, 1967.

19. J. A. Denis and S. J. Bоoт. Dependence of the oxygen enhancement ratio on neutron energy. Nature (London) 215, 310-311 (1967). 
http://www.jstor.org

\title{
LINKED CITATIONS
}

- Page 1 of 2 -

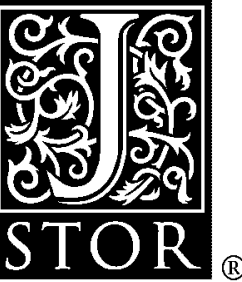

You have printed the following article:

Radiobiological Studies with Monoenergetic Neutrons

E. J. Hall; H. H. Rossi; A. M. Kellerer; L. Goodman; S. Marino

Radiation Research, Vol. 54, No. 3. (Jun., 1973), pp. 431-443.

Stable URL:

http://links.jstor.org/sici?sici=0033-7587\%28197306\%2954\%3A3\%3C431\%3ARSWMN\%3E2.0.CO\%3B2-O

This article references the following linked citations. If you are trying to access articles from an off-campus location, you may be required to first logon via your library web site to access JSTOR. Please visit your library's website or contact a librarian to learn about options for remote access to JSTOR.

\section{References}

\section{${ }^{2}$ Power Relations as an Expression of Relative Biological Effectiveness (RBE) in Tradescantia Stamen Hairs}

A. G. Underbrink; A. H. Sparrow

Radiation Research, Vol. 46, No. 3. (Jun., 1971), pp. 580-587.

Stable URL:

http://links.jstor.org/sici?sici=0033-7587\%28197106\%2946\%3A3\%3C580\%3APRAAEO\%3E2.0.CO\%3B2-T

\section{${ }^{3}$ Relative Biological Effectiveness of X-Rays and 0.43-MeV Monoenergetic Neutrons on Somatic Mutations and Loss of Reproductive Integrity in Tradescantia Stamen Hairs}

A. G. Underbrink; Rhoda C. Sparrow; A. H. Sparrow; H. H. Rossi

Radiation Research, Vol. 44, No. 1. (Oct., 1970), pp. 187-203.

Stable URL:

http://links.jstor.org/sici?sici=0033-7587\%28197010\%2944\%3A1\%3C187\%3ARBEOXA\%3E2.0.CO\%3B2-\%23

\author{
${ }^{10}$ The Recovery of Vicia faba Meristem Cells from X-Radiation \\ Eric J. Hall; Laszlo G. Lajtha \\ Radiation Research, Vol. 20, No. 2. (Oct., 1963), pp. 187-194. \\ Stable URL: \\ http://links.jstor.org/sici?sici=0033-7587\%28196310\%2920\%3A2\%3C187\%3ATROVFM\%3E2.0.CO\%3B2-C
}

NOTE: The reference numbering from the original has been maintained in this citation list. 
http://www.jstor.org

\title{
LINKED CITATIONS \\ - Page 2 of 2 -
}

\author{
${ }^{11}$ RBE and the Primary Mechanism of Radiation Action \\ Albrecht M. Kellerer; Harald H. Rossi \\ Radiation Research, Vol. 47, No. 1. (Jul., 1971), pp. 15-34. \\ Stable URL: \\ http://links.jstor.org/sici?sici=0033-7587\%28197107\%2947\%3A1\%3C15\%3ARATPMO\%3E2.0.CO\%3B2-2 \\ ${ }^{15}$ Dose-Dependence of Fast Neutron RBE for Lens Opacification in Mice \\ J. L. Bateman; H. H. Rossi; A. M. Kellerer; C. V. Robinson; V. P. Bond \\ Radiation Research, Vol. 51, No. 2. (Aug., 1972), pp. 381-390. \\ Stable URL: \\ http://links.jstor.org/sici?sici=0033-7587\%28197208\%2951\%3A2\%3C381\%3ADOFNRF\%3E2.0.CO\%3B2-F
}

NOTE: The reference numbering from the original has been maintained in this citation list. 